\title{
Clonal hematopoiesis in individuals with ANKRD26 or ETV6 germline mutations
}

Michael W. Drazer ${ }^{1,2 *}$, Claire C. Homan ${ }^{3,4 *}$, Kai Yu${ }^{5 *}$, Marcela Cavalcante de Andrade Silva, ${ }^{6}$ Kelsey E. McNeely ${ }^{1}$, Matthew J. Pozsgai ${ }^{1}$, Maria G. Acevedo ${ }^{1}$, Jeremy P. Segal ${ }^{7}$, Peng Wang ${ }^{7}$, Jinghua Feng ${ }^{4}$, Sarah L. King-Smith ${ }^{3,4}$, Erika Kim ${ }^{5}$, Sophia C. Korotev ${ }^{1}$, David M. Lawrence ${ }^{3,4}$, Andreas W. Schreiber ${ }^{3,4,8}$, Christopher N. Hahn ${ }^{3,4,9}$, Hamish S. Scott ${ }^{3,4,9}$, Raman Sood ${ }^{5}$, NISC Comparative Sequencing Program ${ }^{10}$, Elvira D R P Velloso $^{11}$, Anna L. Brown ${ }^{3,4,9}$, Paul P. Liu ${ }^{5}$, and Lucy A. Godley ${ }^{1,2}$

1Section of Hematology/Oncology, University of Chicago, Chicago, Illinois, USA

2Department of Human Genetics, University of Chicago, Chicago, Illinois, USA ${ }^{3}$ Department of Genetics and Molecular Pathology, SA Pathology, Adelaide, SA, Australia ${ }^{4}$ Centre for Cancer Biology, SA Pathology and University of South Australia, Adelaide, SA, Australia

${ }^{5}$ National Human Genome Research Institute, National Institutes of Health, Bethesda, Maryland, USA

${ }^{6}$ Hospital Universitário Prof Alberto Antunes/Universidade Federal de Alagoas, Brazil

${ }^{7}$ Department of Pathology, University of Chicago, Chicago, Illinois, USA

${ }^{8}$ School of Biological Sciences, University of Adelaide, Adelaide, SA, Australia

${ }^{9}$ School of Medicine, University of Adelaide, Adelaide, SA, Australia

${ }^{10} \mathrm{NIH}$ Intramural Sequencing Program, National Human Genome Research Institute, National Institutes of Health, Bethesda, Maryland, USA

${ }^{11}$ Service of Hematology, Transfusion and Cell Therapy and Laboratory of Medical Investigation in Pathogenesis and Directed Therapy in Onco-Immuno-Hematology (LIM31) HCFMUSP, University of Sao Paulo Medical School, Sao Paulo, Brazil; Genetics Laboratory, Hospital Israelita Albert Einstein, Sao Paulo, Brazil.

${ }^{*}$ Contributed equally

Corresponding author:

Lucy A. Godley

Igodley@medicine.bsd.uchicago.edu

5841 S. Maryland Avenue

MC2115

Chicago, IL 606

P: 773.702 .4140

F: 773.702 .9268

Table: 1

Figure: 1

Word count: 1351

References: 15 


\begin{abstract}
Currently, there are at least a dozen recognized hereditary hematopoietic malignancies (HHMs), some of which phenocopy others. Among these, three HHMs driven by germline mutations in ANKRD26, ETV6, or RUNX1 share a phenotype of thrombocytopenia, qualitative platelet defects, and an increased lifetime risk of hematopoietic malignancies (HMs). Prior work has demonstrated that RUNX1 germline mutation carriers experience an elevated lifetime risk $(66 \%)$ for developing clonal hematopoiesis $(\mathrm{CH})$ prior to age 50. Germline mutations in ANKRD26 or ETV6 phenocopy RUNX1 germline mutations, but no studies have focused on the risk of $\mathrm{CH}$ in individuals with germline mutations in ANKRD26 or ETV6.
\end{abstract}

To determine the prevalence of $\mathrm{CH}$ in individuals with germline mutations in ANKRD26 or ETV6, we performed next generation sequencing on hematopoietic tissue from twelve individuals with either germline ANKRD26 or germline ETV6 mutations. Each patient had thrombocytopenia but had not developed HMs. Among the seven individuals with germline $A N K R D 26$ mutations, one patient had a $\mathrm{CH}$ clone driven by a somatic SF3B1 mutation (p.Lys700Glu). This mutation increased from a variant allele frequency (VAF) of $9.4 \%$ at age 56 to $17.4 \%$ at age 60 . None of the germline ETV6 mutation carriers had evidence of $\mathrm{CH}$ at the limits of detection of the NGS assay (5\% VAF). Unlike individuals with germline mutations in $R U N X 1$, no individuals under the age of 50 with germline mutations in ANKRD26 or ETV6 had detectable $\mathrm{CH}$. This work demonstrates that $A N K R D 26$ germline mutation carriers, but not ETV6 mutation carriers, experience elevated risk for $\mathrm{CH}$. 


\section{To the Editor:}

Currently, there are at least a dozen recognized hereditary hematopoietic malignancies (HHMs), some of which phenocopy others. Among these, three HHMs driven by germline mutations in ANKRD26, ETV6, or RUNX1 share a phenotype of thrombocytopenia, qualitative platelet defects, and an increased lifetime risk of hematopoietic malignancies (HMs). ${ }^{1}$ Individuals with germline mutations in these hereditary thrombocytopenia/hereditary hematopoietic malignancy (HT/HHM) associated genes experience a lifetime risk for HMs of approximately 8\% (ANKRD26), 33\% (ETV6), or $44 \%(R U N X 1){ }^{1}$

Nine unaffected RUNX1 germline mutation carriers with thrombocytopenia, but no HMs, were sequenced in a previous study. This demonstrated that $66 \%$ of these individuals had clonal hematopoiesis $(\mathrm{CH})$ prior to age 50 , an elevated $\mathrm{CH}$ risk as compared to population controls. ${ }^{2,3} \mathrm{~A}$ subsequent study of four RUNX1 germline mutation carriers (age 49, 53, 56, and 71 years) with thrombocytopenia, but no HMs, demonstrated $\mathrm{CH}$ in three of these individuals. ${ }^{4}$ Germline mutations in ANKRD26 or ETV6 phenocopy RUNX1 germline mutations, but no studies have focused on the risk of $\mathrm{CH}$ in individuals with germline mutations in ANKRD26 or ETV6.

To address this knowledge gap, we performed a cross sectional study of twelve individuals with either germline ANKRD26 or germline ETV6 mutations who had thrombocytopenia but who had not developed HMs. We determined if ANKRD26 or ETV6 germline mutations lead to increased rates of $\mathrm{CH}$, as is observed in $\mathrm{RUNX1}$ mutation carriers. $^{2,4}$ Given that the penetrance of HMs is lower in ANKRD26 and ETV6 germline mutation carriers than in RUNX1 mutation carriers, we hypothesized that germline 
ANKRD26 or ETV6 mutation carriers would experience lower rates of $\mathrm{CH}$ relative to germline RUNX1 mutation carriers of similar ages. ${ }^{1}$ Additionally, all pathogenic/likely pathogenic ANKRD26 variants with supporting evidence in ClinVar are located in a regulatory domain, the $5^{\prime}$ untranslated region (UTR). ${ }^{5}$ RUNX1 encodes for a transcription factor (RUNX1) that binds to the ANKRD26 5' UTR and suppresses ANKRD26 expression (Supplementary Figure 1). ${ }^{5}$ Therefore, we hypothesized that ANKRD26 and RUNX1 germline mutation carriers would experience somatic mutations in a similar set of genes as compared to ETV6 germline mutation carriers.

We enrolled twelve patients from unrelated families on Institutional Review Boardapproved protocols at the University of Chicago (UChicago) or the Hospital das Clínicas da Faculdade de Medicina da Universidade de São Paulo, Brazil (Supplementary Table 1). Seven unaffected ANKRD26 mutation carriers and four unaffected ETV6 mutation carriers were enrolled. Patient ages ranged from 8 to 63 years. Additionally, we included one affected $A N K R D 26$ mutation carrier with acute myeloid leukemia (AML) with $23 \%$ blasts in order to compare $\mathrm{CH}$-related mutations to an $\mathrm{HM}$ in this syndrome (Table 1, Supplementary Table 1). Supplementary Figure 2 shows a pedigree for each family. Each HT/HHM-related germline variant was classified using Association for Molecular Pathology and American College of Human Genetics and Genomics criteria. ${ }^{6}$ Patient samples included germline tissue (cultured skin fibroblasts) or hematopoietic tissue equivalents (peripheral blood, bone marrow, or saliva). Panel-based sequencing was performed at UChicago as described previously. ${ }^{7}$ Details regarding sample processing and sequencing are in the Supplementary Methods section.

The ages of individuals with germline ANKRD26 or ETV6 mutations at the time of 
sample collection are shown in Table 1. The median age at sample collection was 43 years for unaffected ANKRD26 germline mutation carriers, with serial samples from one individual. The median age for unaffected ETV6 germline mutation carriers was 59 years, with serial samples from two individuals.

Among the seven individuals with germline ANKRD26 mutations, one patient with a germline $A N K R D 26$ mutation had a $\mathrm{CH}$ clone driven by a somatic SF3B1 mutation (p.Lys700Glu). This mutation increased from a variant allele frequency (VAF) of $9.4 \%$ at age 56 to $17.4 \%$ at age 60 (Table 1, Figure 1). SF3B1 p.Lys700Glu is a recognized somatic hotspot mutation that is observed in $2.1 \%$ of COSMIC HMs $(n=525 / 25028) .{ }^{8}$ Of note, the only patient with $\mathrm{CH}$ in the $A N K R D 26$ cohort was also the oldest individual in that cohort. None of the germline ETV6 mutation carriers $(n=4)$ had evidence of $\mathrm{CH}$ at the limits of detection of the NGS assay ( $5 \%$ VAF). Unlike individuals with germline mutations in $R U N X 1$, no individuals under the age of 50 with germline mutations in ANKRD26 $(\mathrm{n}=6)$ or ETV6 $(\mathrm{n}=3)$ had detectable $\mathrm{CH}$ despite nearly half of the unaffected samples being collected from individuals in this age group (Figure 1). ${ }^{2,4}$

The only patient with a germline ANKRD26 mutation and a malignancy (AML) had a leukemic clone with both typical and atypical driver mutations: CUX1 (p. Phe472GInfsX105, VAF 92.4\%), RUNX1 (p.Arg320X, VAF 55.8\%), TET2 (p.Phe1309LeufsX54, VAF 40.0\%), FLT3 (p.Asp835His, second tyrosine kinase domain (TKD), VAF 18.3\%), and SAMD9 (p.Val798GlyfsX7, VAF 13.3\%) (Table 1). FLT3 is mutated in $18.8 \%$ of $\mathrm{COSMIC} \mathrm{HMs}^{8}$ and ${ }^{8}$ UNX1 somatic mutations are the most common second hit in RUNX1 germline mutation carriers who have developed HMs. ${ }^{8,9}$ Given the role of RUNX1 in regulating $A N K R D 26$ expression, the $R U N X 1$ mutation in this 
ANKRD26 germline mutation carrier may effectively represent a second hit that serves as a late leukemogenic event. ${ }^{5}$ The $C U X 1$ and SAMD9 mutations were not described previously in COSMIC. ${ }^{8}$ The leukemic karyotype was 46, XX, -6, del(7)(q11.2),+mar[20].

The total observation time for the ANKRD26 cohort was 275 years. The incidence of $\mathrm{CH}$ in the $A N K R D 26$ cohort was $4.5 \times 10^{-3} \mathrm{CH}$ cases/observation year $(4.5 \mathrm{CH}$ cases per 1000 observation years). The incidence rate of HMs in the ANKRD26 cohort was $3.6 \times 10^{-3}$ malignancies/observation year (3.6 malignancies per 1000 observation years). This HM incidence rate was similar to that seen previously in a cohort of Italian germline ANKRD26 mutation carriers (2.13 malignancies per 1000 observation years). ${ }^{10}$ The observation time for the ETV6 cohort was 258 years, with no diagnoses of $\mathrm{CH}$ or HMs.

Among the known HT/HHM phenocopies, only RUNX1 has been systematically evaluated for $\mathrm{CH}$ risk. This bias has likely occurred for two reasons. First, RUNX1-driven $\mathrm{HT} / \mathrm{HHMs}$ were identified 12 years before ANKRD26-driven HT/HHMs and 16 years before ETV6-driven HT/HHMs, which has provided a longer period of time for researchers to identify and study families with germline RUNX1 mutations. ${ }^{1,11-13}$ Second, RUNX1driven $\mathrm{HT} / \mathrm{HHMs}$ have the highest penetrance for HMs among the HT/HHM phenocopies, with approximately $44 \%$ of mutation carriers developing blood cancers. This penetrance is higher than that experienced by ANKRD26 (8\%) and ETV6 (33\%) germline mutation carriers. ${ }^{1}$ In our clinical experience, the most "severe" hereditary syndromes are more easily recognized than syndromes with more subtle symptoms and lower penetrance phenotypes. Therefore, it is not surprising that prior work in the HHM field has largely focused on RUNX1-driven HT/HHMs. 
To our knowledge, this is the first study examining pre-leukemic states in the HT/HHM phenocopies driven by germline ANKRD26 or ETV6 mutations. In our cohort, $\mathrm{CH}$ was detected in $14 \%$ of $A N K R D 26$ germline mutation carriers, but no $\mathrm{CH}$ was present in ETV6 germline mutation carriers. No ANKRD26 or ETV6 mutation carriers developed malignancies during 533 years of observation time. It is possible the limited number of germline variants and families in this study, with four families carrying three ANKRD26 variants and one family with one ETV6 variant, are not representative of the leukemogenic risk observed in the full spectrum of $\mathrm{HT} / \mathrm{HHM}$-related ANKRD26 or ETV6 germline variants. Ultimately, larger numbers of germline ANKRD26 or ETV6 mutation carriers should be studied to better determine the pre-leukemic genetic milieu that exists in these syndromes.

In conclusion, this is the first cross sectional study focused on leukemogenic mechanisms in individuals with ANKRD26- or ETV6-driven HT/HHM phenocopies. We identified $\mathrm{CH}$ in $14 \%$ of older germline $A N K R D 26$ mutation carriers but did not detect $\mathrm{CH}$ in ETV6 germline mutation carriers. We did not detect early-onset $\mathrm{CH}$ under the age of 50 in individuals with germline ANKRD26 or ETV6 mutations, as has been observed in RUNX1 mutation carriers. ${ }^{2}$ We also identified a rare somatic RUNX1 mutation in a germline ANKRD26 mutation carrier with AML, which may effectively represent a second hit event given the role of RUNX1 in regulating ANKRD26 expression. ${ }^{5}$ Given the relatively small sample size of our cohort and the limited number of pedigrees with germline ANKRD26 mutations worldwide, future studies focused on evaluating leukemogenic mutations before and after the development of HMs in ANKRD26 or ETV6 germline mutation carriers should be performed. 


\section{Acknowledgements}

We thank the patients and their families for their participation in this research program and for providing samples. This work was supported by the Damon Runyon Cancer Research Foundation Physician-Scientist Training Award, the Edward P. Evans Foundation Young Investigator Award, the Cancer Research Foundation Young Investigator Award, and the NIH Paul Calabresi K12 Program in Oncology (MWD). KY, EK, RS, and PPL are supported by the Intramural Research Program at the National Human Genome Research Institute, NIH.

\section{Disclosures}

MWD has received consulting fees from Cardinal Health, Inc. HSS has received honoraria from Celgene, Inc. LAG receives royalties from a coauthored article on inherited hematopoietic malignancies in UpToDate, Inc. 
bioRxiv preprint doi: https://doi.org/10.1101/2021.11.17.468983; this version posted November 22, 2021. The copyright holder for this preprint (which was not certified by peer review) is the author/funder, who has granted bioRxiv a license to display the preprint in perpetuity. It is made available under aCC-BY-NC-ND 4.0 International license.

\section{Tables and Figures}

Table 1. Germline mutations and somatic driver mutations identified in each individual in ANKRD26 and ETV6-mutated HT/HHM phenocopy cohort. Family numbers and Individual IDs reference pedigrees shown in Supplementary Figure 2.

\begin{tabular}{|c|c|c|c|c|c|c|c|}
\hline \multicolumn{8}{|c|}{ Unaffected Individuals } \\
\hline Germline Gene & Variant & Family & Individual ID & Age & CH present? & CH mutation & VAF \\
\hline ANKRD26 & 5' UTR c.-128G>A NM_014915.2 & 4 & III-16 & 8 & $\mathrm{~N}$ & NA & NA \\
\hline ANKRD26 & 5' UTR c.-128G>A NM_014915.2 & 4 & III-14 & 22 & $\mathrm{~N}$ & NA & NA \\
\hline ANKRD26 & 5' UTR c.-118C>T NM_014915.2 & 2 & IV-1 & 25 & $\mathrm{~N}$ & NA & NA \\
\hline$A N K R D 26$ & 5' UTR c.-118C>T NM_014915.2 & 2 & III-4 & 55 & $\mathrm{~N}$ & NA & NA \\
\hline$A N K R D 26$ & 5' UTR c.-118C>T NM_014915.2 & 2 & III-2 & 56 & $\mathrm{Y}$ & SF3B1 p.Lys700Glu & $9.4 \%$ \\
\hline$A N K R D 26$ & 5' UTR c.-118C>T NM_014915.2 & 2 & III-2 & 60 & $\mathrm{Y}$ & SF3B1 p.Lys700Glu & $17.4 \%$ \\
\hline ANKRD26 & 5' UTR c.-119C>G NM_014915.2 & 3 & IV-3 & 43 & $\mathrm{~N}$ & NA & NA \\
\hline$A N K R D 26$ & 5' UTR c.-119C>G NM_014915.2 & 3 & IV-3 & 44 & $\mathrm{~N}$ & NA & NA \\
\hline ANKRD26 & 5' UTR c.-119C>G NM_014915.2 & 3 & $V-1$ & 13 & $\mathrm{~N}$ & NA & NA \\
\hline ETV6 & p.R369Q NM_001987.4 & 1 & IV-5 & 33 & $\mathrm{~N}$ & NA & NA \\
\hline ETV6 & p.R369Q NM_001987.4 & 1 & IV-4 & 36 & $\mathrm{~N}$ & NA & NA \\
\hline ETV6 & p.R369Q NM_001987.4 & 1 & IV-4 & 38 & $\mathrm{~N}$ & NA & NA \\
\hline ETV6 & p.R369Q NM_001987.4 & 1 & III-3 & 59 & $\mathrm{~N}$ & NA & NA \\
\hline ETV6 & p.R369Q NM_001987.4 & 1 & III-3 & 62 & $\mathrm{~N}$ & NA & NA \\
\hline ETV6 & p.R369Q NM_001987.4 & 1 & III-5 & 62 & $\mathrm{~N}$ & NA & NA \\
\hline ETV6 & p.R369Q NM_001987.4 & 1 & III-3 & 63 & $\mathrm{~N}$ & NA & NA \\
\hline \multicolumn{8}{|l|}{ Affected Individuals } \\
\hline Germline Gene & Variant & & Individual ID & Age & Malignancy & Somatic Driver Mutations & VAF \\
\hline \multirow[t]{5}{*}{$A N K R D 26$} & 5' UTR c.-128G>A NM_014915.2 & 4 & II-12 & 48 & AML ( $23 \%$ blasts) & CUX1 p. Phe472GInfsX105 & $92.4 \%$ \\
\hline & & & & & & RUNX1 p.Arg320X & $55.8 \%$ \\
\hline & & & & & & TET2 p.Phe1309LeufsX54 & $40.0 \%$ \\
\hline & & & & & & FLT3 p.Asp835His & $18.3 \%$ \\
\hline & & & & & & SAMD9 p.Val798GlyfsX7 & $13.3 \%$ \\
\hline
\end{tabular}


Figure 1. Clonal hematopoiesis in ANKRD26 or ETV6 germline mutation carriers. One individual with a germline ANKRD26 mutation (5' UTR, c.-118C>T, NM_014915.2) had a CH clone driven by SF3B1 p.Lys700Glu in two samples collected at the ages of 56 and 60.

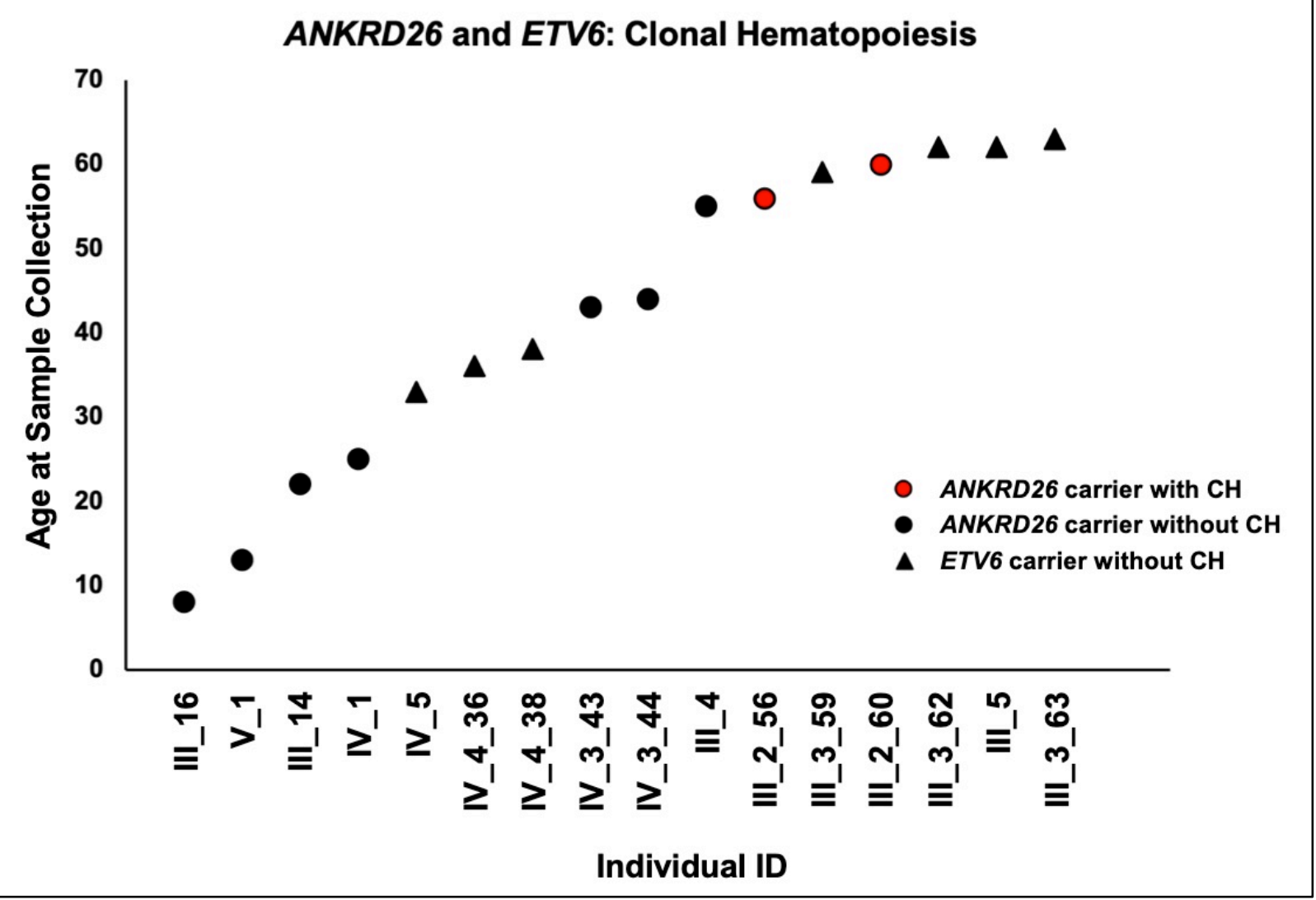




\section{Supplementary Files}

Supplementary Table 1. Cohort of patients enrolled on the ANKRD26 and ETV6 cross sectional study.

\begin{tabular}{|c|c|c|c|c|c|}
\hline Germline Gene of Interest & Variants & Individual & Family & Phenotype & Age(s) at Sample Collection (years) \\
\hline ETV6 & p.R369Q NM_001987.4 & III_3 & Family 1 & Thrombocytopenia & $59,62,63$ \\
\hline ETV6 & p.R369Q NM_001987.4 & IV_4 & Family 1 & Thrombocytopenia & 36,38 \\
\hline ETV6 & p.R369Q NM_001987.4 & IV_5 & Family 1 & Thrombocytopenia & 33 \\
\hline ETV6 & p.R369Q NM_001987.4 & III_5 & Family 1 & Thrombocytopenia & 62 \\
\hline ANKRD26 & 5' UTR c.-118C>T NM_014915.2 & III_2 & Family 2 & Thrombocytopenia & 56,60 \\
\hline ANKRD26 & 5' UTR c.-118C>T NM_014915.2 & III_4 & Family 2 & Thrombocytopenia & 55 \\
\hline ANKRD26 & 5' UTR c.-118C>T NM_014915.2 & IV_1 & Family 2 & Thrombocytopenia & 25 \\
\hline ANKRD26 & 5' UTR c.-119C>G NM_014915.2 & IV_3 & Family 3 & Thrombocytopenia & 43,44 \\
\hline ANKRD26 & 5' UTR c.-119C>G NM_014915.2 & V_1 & Family 3 & Thrombocytopenia & 13 \\
\hline ANKRD26 & 5' UTR c.-128G>A NM_014915.2 & III_14 & Family 4 & Thrombocytopenia & 22 \\
\hline ANKRD26 & 5' UTR c.-128G>A NM_014915.2 & II_12 & Family 4 & AML ( $23 \%$ blasts) & 48 \\
\hline ANKRD26 & 5' UTR c.-128G>A NM_014915.2 & III 16 & Family 4 & Thrombocytopenia & 8 \\
\hline
\end{tabular}

Supplementary Figure 1. The 5' UTR region of ANKRD26 contains a binding site for both RUNX1 and FLI1. Germline mutations in the 5' UTR of ANKRD26 disrupt this interaction and lead to a hereditary thrombocytopenia/hereditary hematopoietic malignancy (HT/HHM) phenotype. This phenotype phenocopies germline RUNX1 mutations. Germline FLI1 mutations lead to hereditary thrombocytopenia but are not known to cause HHMs and are therefore not considered to represent an HT/HHM phenocopy. The mutations shown (c.$128 \mathrm{G}>\mathrm{A}, \mathrm{c} .-119 \mathrm{C}>\mathrm{G}$, and c. $-118 \mathrm{C}>\mathrm{T}$ ) represent the mutations in the cohort of ANKRD26 germline mutation patients in this study. ClinVar classifies the c. $-118 \mathrm{C}>\mathrm{T}$ and $\mathrm{C} .-119 \mathrm{C}>\mathrm{G}$ variants as likely pathogenic and the $\mathrm{c} .-128 \mathrm{G}>\mathrm{A}$ variant as pathogenic.

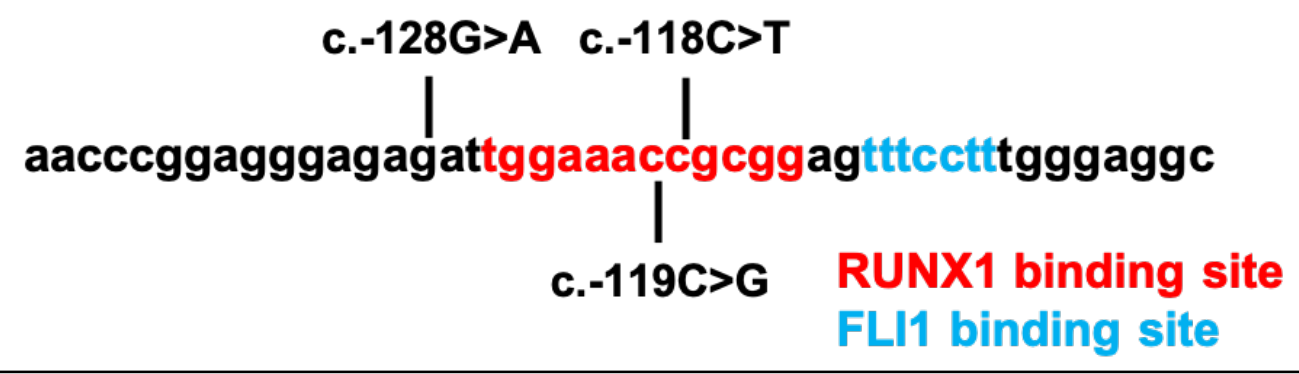


bioRxiv preprint doi: https://doi.org/10.1101/2021.11.17.468983; this version posted November 22, 2021. The copyright holder for this preprint (which was not certified by peer review) is the author/funder, who has granted bioRxiv a license to display the preprint in perpetuity. It is made available under aCC-BY-NC-ND 4.0 International license.

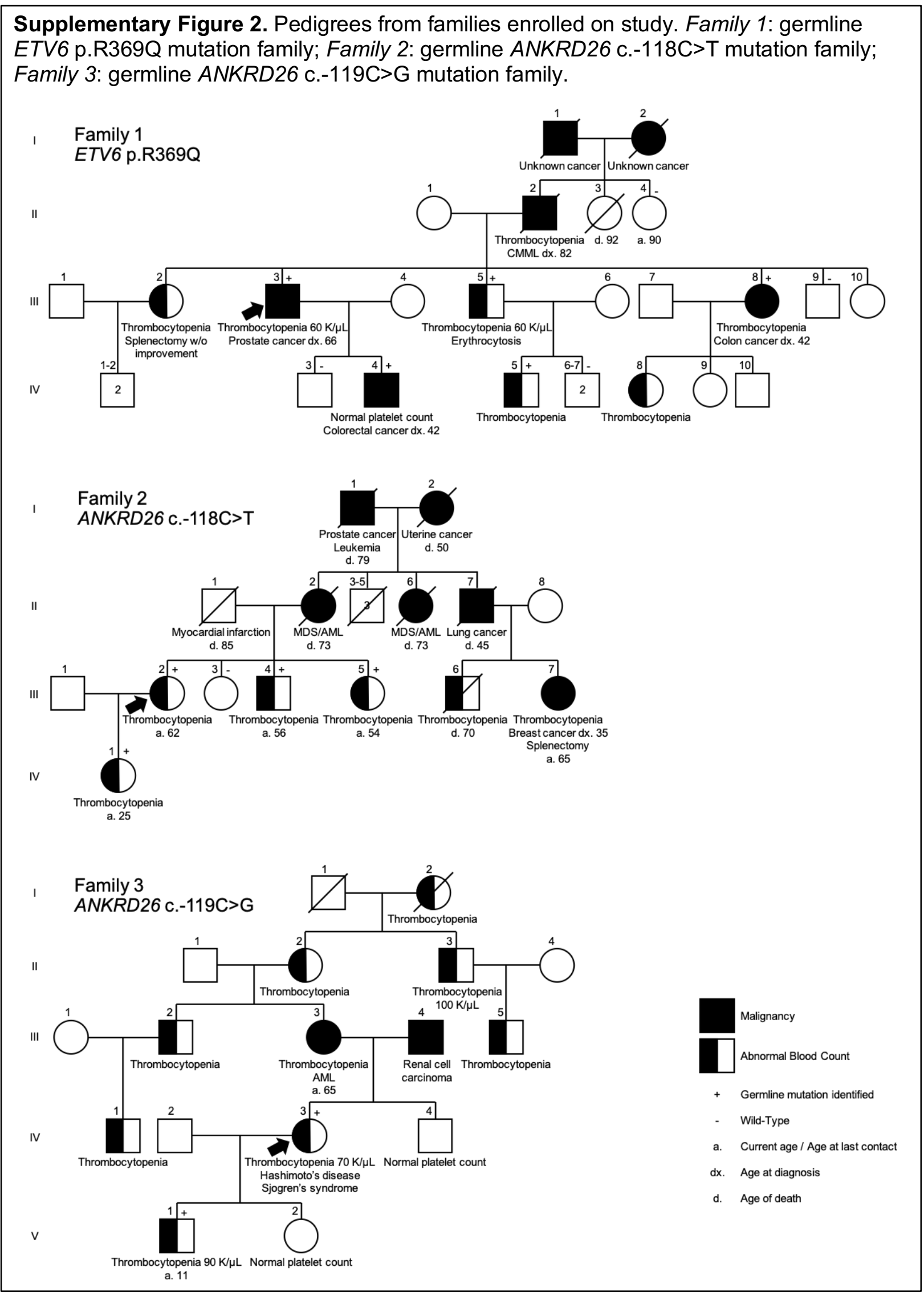




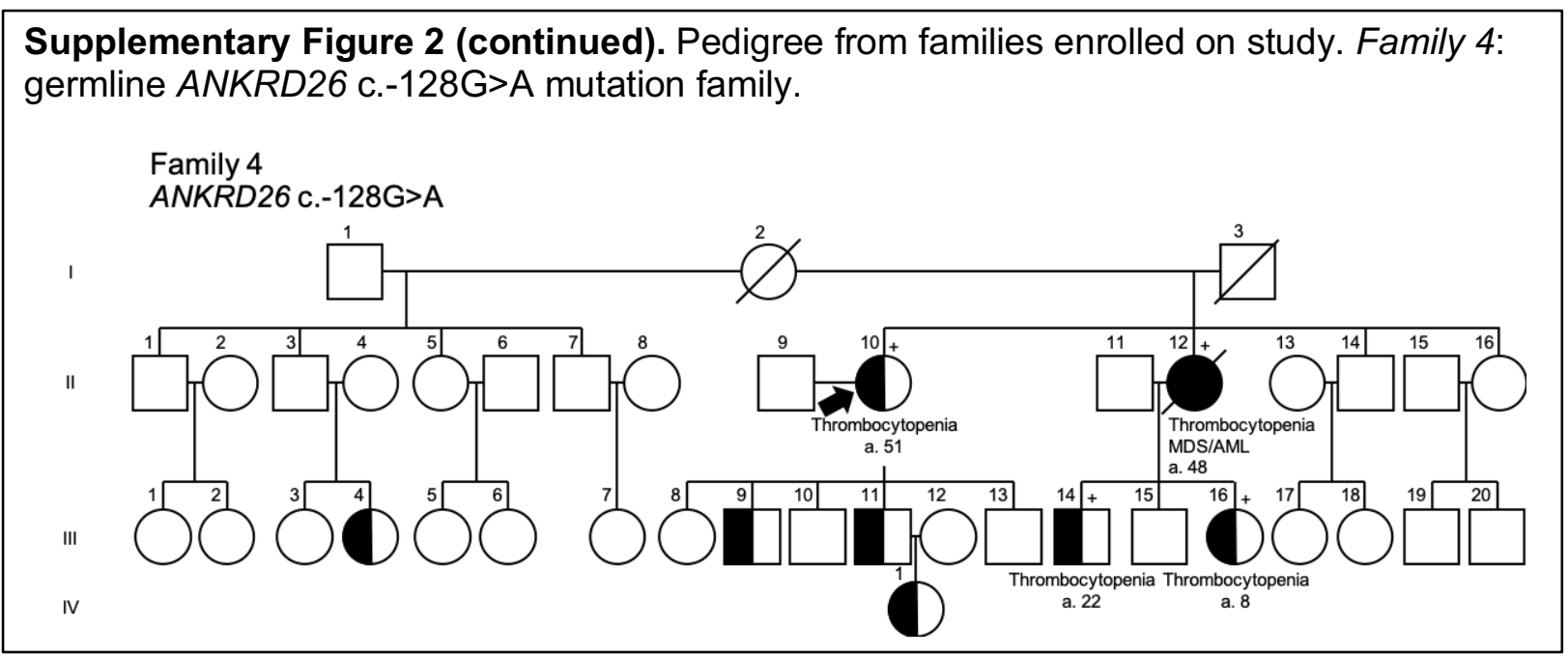

\section{Supplementary Methods}

Sample processing and next generation sequencing methods

Genomic DNA (gDNA) was extracted with the QIAamp DNA Blood Mini Kit (Qiagen) following the manufacturer's instructions. DNA concentrations were measured via a Nanodrop (Thermo Scientific) and/or Qubit fluorometer (Life Technologies). At least 100 ng of genomic DNA from each sample was sheared, selected by size, ligated to adapters, and standard sequencing libraries were generated via PCR amplification. Following library generation, genomic capture was performed using a custom SeqCap EZ capture panel that covered 1212 genes (Roche), and an additional PCR amplification with realtime quantitative PCR quantification was performed. An Illumina HiSeq was used to sequence the pooled capture libraries. Sequencing data were stored on a protected highperformance computing system at UChicago that exceeds requirements for the Health Insurance Portability and Accountability Act. The data were initially analyzed via a bioinformatics pipeline that melded publicly available packages built off of the GATK package and a custom bioinformatics pipeline developed at UChicago. These data were 
initially reviewed by MWD and KY for driver mutations. ${ }^{14}$ Following an initial round of review, the raw FASTQ files were then transferred to the University of South Australia in order to analyze the data using the freebayes-based RUNX1db bioinformatics pipeline. ${ }^{15}$ The data were filtered for read quality and depth as previously described, with thresholds as follows: variant allelic depth $>=5$, read depth $>=20$, population prevalence (variants at $0.1 \%$ or higher in any population database were removed), pathogenicity (missense variants that were not predicted to be damaging in 2 or more in silico predictors were removed; CADD scores with values less than 20 or higher were removed), and oncogenicity (variants not in genes with known roles as drivers in myeloid malignancies, not in COSMIC, or RUNX1 variants were removed). We analyzed the subsequent list of candidate variants and used IGV to review each variant of interest manually in the individual BAM files. We removed any variants labeled as artifacts after the aforementioned steps.

We then analyzed the remaining IGV-confirmed variants to label each variant as germline or somatic in origin. For individuals with sequencing data from cultured skin fibroblasts, we compared variants identified in hematopoietic tissue equivalents directly to data obtained from cultured skin fibroblasts. Samples without paired germline tissue were analyzed using a combination of population allelic frequency (minor allele threshold of $0.01 \%$ or lower), VAF (with likely germline VAFs considered to be between 30 and $60 \%$ for genes on autosomal chromosomes and $80 \%$ or higher for genes on the $X$ chromosome), and the frequency of the variant in question in tumor databases such as COSMIC. Any variant passing the above population filters, but which still occurred more than twice in COSMIC, was considered to not be of germline origin. 
This filtering process produced a list of variants of likely somatic or definitive somatic origin which we reviewed manually for clinical and biological relevance. The determination of "likely somatic" or "somatic" origin adhered to criteria defined in the original RUNX1 database manuscript. ${ }^{15}$ "Clinically relevant" variants were known pathogenic germline variants in leukemia or variants that were present more than twice in COSMIC in hematopoietic and lymphoid samples (H\&L samples). Novel driver variants were clinically relevant if they were present in a gene known to be recurrently mutated in COSMIC H\&L samples, were a truncating variant (nonsense, frameshift indels, essential splice site variants), were in the same domain as known pathogenic variants (for example, the RUNT domain in RUNX1), or were a deletion in a gene where deletion is a known mechanism of disease. Missense variants were considered to be clinically relevant if they were damaging in at least 3 in silico algorithms and were highly conserved via GERP and Phylop scores. All somatic and likely somatic variants that did not meet criteria for clinical relevance were categorized as "possibly relevant" or "of unknown relevance". 


\section{References}

1. Feurstein S, Drazer MW, Godley LA. Genetic predisposition to leukemia and other hematologic malignancies. Semin Oncol 2016 Oct; 43(5): 598-608.

2. Churpek JE, Pyrtel K, Kanchi KL, Shao J, Koboldt D, Miller CA, et al. Genomic analysis of germline and somatic variants in familial myelodysplasia/acute myeloid leukemia. Blood 2015 Oct.

3. Genovese G, Kähler AK, Handsaker RE, Lindberg J, Rose SA, Bakhoum SF, et al. Clonal hematopoiesis and blood-cancer risk inferred from blood DNA sequence. N Engl J Med 2014 Dec; 371(26): 2477-2487.

4. DiFilippo EC, Coltro G, Carr RM, Mangaonkar AA, Binder M, Khan SP, et al. Spectrum of abnormalities and clonal transformation in germline RUNX1 familial platelet disorder and a genomic comparative analysis with somatic RUNX1 mutations in MDS/MPN overlap neoplasms. Leukemia 2020 09; 34(9): 2519-2524.

5. Bluteau D, Balduini A, Balayn N, Currao M, Nurden P, Deswarte C, et al. Thrombocytopenia-associated mutations in the ANKRD26 regulatory region induce MAPK hyperactivation. J Clin Invest 2014 Feb; 124(2): 580-591.

6. Richards S, Aziz N, Bale S, Bick D, Das S, Gastier-Foster J, et al. Standards and guidelines for the interpretation of sequence variants: a joint consensus recommendation of the American College of Medical Genetics and Genomics and the Association for Molecular Pathology. Genet Med 2015 May; 17(5): 405-424.

7. Kadri S, Long BC, Mujacic I, Zhen CJ, Wurst MN, Sharma S, et al. Clinical Validation of a Next-Generation Sequencing Genomic Oncology Panel via CrossPlatform Benchmarking against Established Amplicon Sequencing Assays. $J \mathrm{Mol}$ Diagn 2017 Jan; 19(1): 43-56.

8. Tate JG, Bamford S, Jubb HC, Sondka Z, Beare DM, Bindal N, et al. COSMIC: the Catalogue Of Somatic Mutations In Cancer. Nucleic Acids Res 2019 Jan; 47(D1): D941-D947.

9. Brown AL, Arts P, Carmichael CL, Babic M, Dobbins J, Chong CE, et al. RUNX1mutated families show phenotype heterogeneity and a somatic mutation profile unique to germline predisposed AML. Blood Adv 202003 24; 4(6): 1131-1144.

10. Noris P, Favier R, Alessi MC, Geddis AE, Kunishima S, Heller PG, et al. ANKRD26-related thrombocytopenia and myeloid malignancies. Blood $2013 \mathrm{Sep}$; 122(11): 1987-1989. 
11. Noris P, Perrotta S, Seri M, Pecci A, Gnan C, Loffredo G, et al. Mutations in ANKRD26 are responsible for a frequent form of inherited thrombocytopenia: analysis of 78 patients from 21 families. Blood 2011 Jun; 117(24): 6673-6680.

12. Noetzli L, Lo RW, Lee-Sherick AB, Callaghan M, Noris P, Savoia A, et al. Germline mutations in ETV6 are associated with thrombocytopenia, red cell macrocytosis and predisposition to lymphoblastic leukemia. Nat Genet 2015 May; 47(5): 535538.

13. Zhang MY, Churpek JE, Keel SB, Walsh T, Lee MK, Loeb KR, et al. Germline ETV6 mutations in familial thrombocytopenia and hematologic malignancy. Nat Genet 2015 Feb; 47(2): 180-185.

14. McKenna A, Hanna M, Banks E, Sivachenko A, Cibulskis K, Kernytsky A, et al. The Genome Analysis Toolkit: a MapReduce framework for analyzing nextgeneration DNA sequencing data. Genome Res 2010 Sep; 20(9): 1297-1303.

15. Homan CC, King-Smith SL, Lawrence DM, Arts P, Feng J, Andrews J, et al. The RUNX1 Database (RUNX1db): establishment of an expert curated RUNX1 registry and genomics database as a public resource for familial platelet disorder with myeloid malignancy. Haematologica 2021 Jul. 